\title{
Egg consumption, serum total cholesterol concentrations and coronary heart disease incidence: Japan Public Health Center-based prospective study
}

\author{
Yasuyuki Nakamura ${ }^{1}$, Hiroyasu Iso ${ }^{2}$, Yoshikuni Kita ${ }^{3}$, Hirotsugu Ueshima ${ }^{3}$, Katsutoshi Okada ${ }^{4}$, \\ Masafumi Konishi ${ }^{4}$, Manami Inoue ${ }^{5}$ and Shoichiro Tsugane ${ }^{5}$ for the Japan Public Health Center-based \\ prospective study group \\ ${ }^{1}$ Cardiovascular Epidemiology, Faculty of Home Economics, Kyoto Women's University, 35 Imakumano Kitahiyoshi-cho, \\ Higashiyama-ku, Kyoto 605-8501, Japan \\ ${ }^{2}$ Department of Public Health Medicine, Major of Social and Environmental Medicine, Doctoral Program in Comprehensive \\ Human Sciences, University of Tsukuba, Tsukuba, Japan \\ ${ }^{3}$ Department of Health Science, Shiga University of Medical Science, Otsu, Japan \\ ${ }^{4}$ Department of Public Health, Ehime University School of Medicine, Touon, Japan \\ ${ }^{5}$ Epidemiology and Prevention Division, Research Center for Cancer Prevention and Screening, National Cancer Center, \\ Tokyo, Japan
}

(Received 21 December 2005 - Revised 20 July 2006 - Accepted 20 July 2006)

\begin{abstract}
Limited egg consumption is often recommended to reduce serum cholesterol concentration for the prevention of CHD. We examined the association of egg consumption and total cholesterol concentration with the risk of CHD. A total of 90735 subjects (19856 men and 21408 women, aged 40-59 years in cohort I; 23463 men and 26008 women, aged 40-69 years in cohort II) were followed from 1990-4 to the end of 2001 under the Japan Public Health Center-based prospective study. Total cholesterol was obtained in $36 \%$ of the subjects. Men and women were combined for the analyses. The subjects were categorised into four groups according to egg consumption. Subjects with total cholesterol $\geq 2200 \mathrm{mg} / \mathrm{l}$ were less frequent in frequent egg consumption groups in both cohorts (trend $P<0.0001$ ). Subjects with $<1 \mathrm{~d} /$ week of egg consumption were more likely to avoid a cholesterol-rich diet. Egg consumption was not associated with the risk of CHD, although total cholesterol was significantly related to the risk of CHD. The multivariate hazard ratio of CHD in subjects with total cholesterol $\geq 2400 v$. $<1800 \mathrm{mg} / 1$ was $2 \cdot 17$ (95\% CI 1.22, $3 \cdot 85$; trend $P=0$.0018). In conclusion, eating eggs more frequently, up to almost daily, was not associated with an increase in CHD incidence for middle-aged Japanese men and women. Subjects with hypercholesterolaemia were less frequently in frequent egg consumption groups, probably because they avoided eating eggs.
\end{abstract}

Cholesterol: Coronary disease: Risk factors: Epidemiology

Since eggs are a concentrated source of cholesterol in the diet, limited egg consumption is often recommended to reduce total serum cholesterol concentrations and to help prevent CHD (Expert Panel on Detection, Evaluation, and Treatment of High Blood Cholesterol in Adults, 2001). While several metabolic-ward studies have demonstrated that dietary cholesterol is one of the major determinants of total cholesterol (Mattson et al. 1972; Keys, 1984), other studies have failed to show changes in total cholesterol when eggs were added to diets that already contained moderate amounts of cholesterol (Kummerow et al. 1977; Porter et al. 1977; Flynn et al. 1979; Oh \& Miller, 1985). There have been few epidemiological studies in free-living populations that explored the relationship between egg consumption and total cholesterol and/or risk of CHD, and none of the studies found an association between egg consumption and CHD incidence (Nichols et al. 1976a,b; Frank et al. 1978; Dawber et al. 1982; Hu et al. 1999). A study by
Hu et al. (1999) with 117933 subjects in the USA showed no relationship between egg consumption of up to one per $d$ and the risk of CHD or stroke. However, in geographic areas where egg consumption makes a greater contribution to total dietary cholesterol intake than in the USA, the results may be different (Ueshima et al. 1982; Okayama et al. 1995; Yoshida et al. 1998). In fact, a recent study using the database of NIPPON DATA80, which included more than 10000 randomly selected subjects in Japan, found a dose-response relationship between egg consumption and total cholesterol in women, but not in men. We also found statistically non-significant tendencies for a higher mortality due to CHD with an increase in egg consumption in women with a 14-year followup (Nakamura et al. 2004). Thus, at least a study in Japan, where egg consumption makes a greater contribution to total dietary cholesterol intake than in Western populations, showed that egg consumption was related to total cholesterol 
concentration in women, but was not associated with a higher mortality due to CHD.

The purpose of the present study was to examine the association between egg consumption and total cholesterol concentration, and CHD incidence in a larger number of subjects.

\section{Subjects and methods}

\section{Study population}

The Japan Public Health Center-based prospective study on cancer and CVD commenced in 1990 for the first group (cohort I) and in 1993 for the second group (cohort II). Cohort I covered four areas administered by public health centres in four prefectures (Iwate, Akita, Nagano and Okinawa). Cohort II included five public health centre areas in five prefectures (Ibaraki, Niigata, Kochi, Nagasaki and Okinawa). Cohort I was comprised of all residents aged 40-59 years on 1 January 1990 and cohort II was comprised of all residents aged 40-69 years on 1 January 1993. The study subjects were identified by population registries maintained by local municipalities. We defined a populationbased cohort of 54512 subjects in cohort I (27439 men and 27073 women) and 62415 subjects in cohort II (31 750 men and 30665 women). A total of 162 subjects in cohort I and 127 subjects in cohort II were excluded because of the following reasons: being non-Japanese, those who had already moved away at baseline, and those who were outside of the age parameters. This left 54350 eligible subjects in cohort I and 62288 eligible subjects in cohort II. The present study was approved by the Institutional Review Board of the National Cancer Center (Tokyo, Japan). The study design is described in detail elsewhere (Watanabe et al. 2001).

\section{Baseline survey}

A self-administered questionnaire was distributed mostly by hand and partly by mail to the study subjects in 1990 for cohort I and in 1993-4 for cohort II. They were asked about their personal and familial medical histories, smoking, alcohol consumption, dietary habits and other lifestyle factors (Tsugane \& Sobue, 2001; Tsugane et al. 2001; Sobue et al. 2001). Among the eligible subjects, 43160 subjects in cohort I (overall 79\%; $76 \%$ men and $82 \%$ women) and 52271 subjects in cohort II (overall 84\%; $81 \%$ men and $86 \%$ women) returned the questionnaire. From them, we excluded subjects with a self-reported medical history of myocardial infarction, IHD, stroke or cancer before the survey began (1555 subjects in cohort I and 2032 subjects in cohort II). This additionally reduced the number of eligible subjects to 41605 subjects in cohort I and 50239 subjects in cohort II. Finally, we excluded subjects with no egg consumption data (341 subjects in cohort I and 768 subjects in cohort II), leaving 90735 subjects (19856 men and 21408 women in cohort I, and 23463 men and 26008 women in cohort II) as study subjects.

The average frequency of egg consumption was reported in four categories in cohort I: 'less than $1 \mathrm{~d} /$ week', ' $1-2 \mathrm{~d} /$ week', ' $3-4 \mathrm{~d} /$ week' and 'almost every day'. Subjects in cohort II were asked about the average frequency of egg consumption divided into five categories: 'never', 'less than $1 \mathrm{~d} /$ week', '1-2d/week', '3-4d/week', and 'almost every day'. Categories: 'never' and 'less than $1 \mathrm{~d}$ /week' in cohort II were combined into the one category, resulting in four categories, the same as those of cohort I. The reproducibility of egg consumption, 5 years apart in cohort I, was measured with the Spearman rank correlation in ninety-four men and 107 women (correlation coefficient was 0.29 for men and 0.40 for women) (Tsubono et al. 2003), and that of 1 year apart in cohort II in 143 men and 146 women (correlation coefficient was 0.50 for men and 0.53 for women) (Ishihara et al. 2003). The validity of egg consumption was estimated with the Spearman rank correlation, with egg consumption based on four $7 \mathrm{~d}$ direct diet records among ninety-four men and 107 women in cohort I (correlation coefficient 0.25 for men and 0.28 for women) (Tsubono et al. 2003), and that in cohort II in 174 men and 176 women (correlation coefficient 0.47 for men and 0.45 for women) (Ishihara et al. 2003).

The frequency of weekly intake of the other twenty-six food items was reported under four categories: 'rarely', ' $1-2 \mathrm{~d} /$ week', '3-4d/week' and 'almost every day'. The weekly frequency for each food item was calculated according to a score assigned to each frequency category $(0,1.5,3.5$ and 7 , respectively).

Also, the subjects were asked whether they were intentionally restricting their intake of certain kinds of foods, for example, in cohort I, 'Do you intend to avoid cholesterolrich diets?'. In cohort II, the questions were more specific, for example, 'Do you intend to avoid cholesterol-rich diets, such as eggs and fat-rich meat?'.

Smoking status was defined as never smoker, ex-smoker and current smoker, and alcohol consumption was classified into six categories in men: 'non-drinkers' $(<1 \mathrm{~d} /$ month $)$, 'occasional drinkers' (1-3d/month), 'weekly alcohol consumption of 1-149 g/week', '150-299 g/week', '300-449 g/ week' and ' $\geq 450 \mathrm{~g} /$ week'. The algorithm for calculating the amount of alcohol has been reported previously (Iso et al. 2004).

\section{Serum total cholesterol concentration data}

The serum total cholesterol concentration data were obtained in subjects who participated in a health check-up programme conducted by each local government. Among the study subjects, health check-up data were obtained in $29 \%$ of men and $46 \%$ of women in cohort I, and $25 \%$ of men and $45 \%$ of women in cohort II. Total cholesterol concentration was analysed enzymically. With regard to the precision and accuracy of the measurement of total cholesterol, all twenty-three laboratories that participated in the present investigation were approved by the Osaka Medical Center for Health Science and Promotion (Japan), a member of the Cholesterol Reference Method Laboratory Network (Myers et al. 2000; Nakamura et al. 2003).

Hypercholesterolaemia was defined as subjects with total cholesterol concentration $\geq 2200 \mathrm{mg} / \mathrm{l}$.

\section{Follow-up and end-point ascertainment}

We followed study subjects until 31 December 2001. The end point for the present study was the incidence of CHD (including CHD death and non-fatal myocardial infarction). Medical 
records were reviewed by registered hospital workers or the study physicians, blinded to the lifestyle data. CHD events were registered if they occurred after the date of return of the baseline questionnaire and before 1 January 2002 .

The criteria of acute myocardial infarction were based on WHO MONICA Projects (Monitoring of Trends and Determinants in CVD): symptoms plus either diagnostic electrocardiogram changes or elevated cardiac enzymes (Tunstall-Pedoe et al. 1994). For complete surveillance of non-fatal myocardial infarction, we asked the possible cases who reported a history of myocardial infarction in a 5-year and 10-year follow-up questionnaire, but had not been registered as a case, by letter or telephone about the onset of myocardial infarction and for permission to review their medical records. Nonfatal myocardial infarction for which confirmatory information was obtained by letter or telephone, but for which no medical records were available, were regarded as probable $(8 \%$ of non-fatal myocardial infarctions).

To complete the surveillance for fatal CHD, we also conducted a systematic search for death certificates. The underlying causes of death were coded according to the 9th International Classification of Disease for the National Vital Statistics (known as ICD-9) and until the end of 1994, and according to the 10th International Classification of Disease (known as ICD-10) from the beginning of 1995. For all fatal CHD listed on the death certificate, but which had not been registered, medical records in registered hospitals were reviewed by hospital workers, the study physicians, or research physicians-epidemiologists. When no medical records were available (death certificate information only), we regarded these fatal CHD as probable CHD (36\% of fatal CHD).

\section{Statistical analysis}

SAS version 8.02 for Windows (SAS Institute, Cary, NC, USA) was used throughout the analyses. Men and women were analysed together in cohort I and cohort II so that the number of cases was sufficient for the analyses. The $\chi^{2}$ test was used to compare dichotomous variables, and a one-way ANOVA was used to compare means among the four groups according to egg consumption. The Mantel-Haenszel $\chi^{2}$ statistical test was used to detect deviation from linearity in the association between nominal variables and the categories of egg consumption, and the ANOVA was used to detect deviation from linearity in the association between continuous variables and the categories of egg consumption. Person-years of follow-up were determined from 1 January 1990 (cohort I) or 1993-4 (cohort II) until the date of a subject's CHD event, the date of moving from a public health centre area, or 31 December 2001, whichever occurred first.

To examine the association between egg consumption and CHD incidence, we calculated the age and sex- and multivariate-adjusted hazard ratios for CHD incidence using a Cox's proportional hazard model, taking the highest egg consumption group, namely almost daily, as the reference (Cox, 1972). For multivariate analyses, age at baseline, sex, BMI $\left(\mathrm{kg} / \mathrm{m}^{2}\right)$, diabetes defined as subjects with a self-reported diagnosis of diabetes mellitus and/or subjects who were taking diabetes-treating medications, hypertension defined as subjects with a selfreported diagnosis and/or subjects who were taking anti-hypertensive medications, cigarette smoking (never, ex-smoker and current smoker), alcohol intake (six categories), use of cholesterol-lowering drugs, dietary intake categories of meat (beef and pork), fish (fresh and dry), vegetables (green, yellow and others), fruits, whether or not intended to avoid cholesterolrich diets, and cohort effects were entered as covariates. Tests of linear trends across groups were conducted by assigning an ordinal value from 1 to 4 for each level of consumption and modelling this as a continuous variable in separate Cox proportional hazard models.

The secondary analyses were performed in cohort I and II separately, in self-reported diabetic patients, in subjects excluding those who had an intension to restrict cholesterol-rich diets, and in subjects excluding those who taking cholesterol-lowering drugs or those with total cholesterol concentration $\geq 2200 \mathrm{mg} / \mathrm{l}$. Furthermore, analysis was performed using quantitative variables (cholesterol and systolic blood pressure) in those who participated in a health check-up programme.

To examine the association between total cholesterol concentration and CHD incidence, we divided the subjects into five categories according to total cholesterol concentration: ' $<1800$ ', '1800-1999', '2000-2199', '2200-2399' and ' $\geq 2400$ ' mg/l, and we calculated the age- and sex-adjusted and multivariateadjusted hazard ratios for CHD incidence using a Cox's proportional hazard model, taking the total cholesterol concentration $<1800 \mathrm{mg} / \mathrm{l}$ group as a reference. For multivariate analyses, we adjusted for age, sex, BMI, systolic blood pressure, diabetes, uses of cholesterol-lowering drugs or anti-hypertensive drugs, smoking (never, ex-smoker and current smoker), alcohol drinking (six categories), whether or not intended to avoid cholesterol-rich diets, consumption frequencies of egg, meat, fish, vegetables, fruits, and cohort effects.All $P$ values $<0.05$ were considered significant. Data are presented as the mean values and SD, unless stated otherwise.

\section{Results}

\section{Baseline characteristics}

Baseline characteristics in each egg consumption category are shown in Table 1. There were more men and thus current smokers in the $1-2 \mathrm{~d} /$ week egg consumption groups (trend $P=0.0001$ and $P<0.0001)$, the reason for which is not clear. The mean age in the less than $1 \mathrm{~d} /$ week egg consumption group was higher (trend $P<0 \cdot 0001$ ). Weekly drinkers were less frequent in the less than $1 \mathrm{~d} /$ week egg consumption group and hypertension was more frequent in the less-frequent egg consumption groups (trend $P<0.0001$ for both). Cholesterol-lowering drugs were more frequently taken in the lessfrequent egg consumption groups (trend $P<0 \cdot 0001$ ).

A significant trend was noted on the information regarding the intention to avoid a cholesterol-rich diet (trend $P<0 \cdot 0001)$. Namely, the fewer eggs consumed, the more frequent the subjects had an intention of avoiding taking a cholesterol-rich diet. Dietary intake of meat (beef and pork), fish (fresh and dry), vegetables (green, yellow and others) and fruits were more frequent among those who in the more-frequent egg consumption groups (all trends $P<0 \cdot 0001$ ).

Data for total cholesterol concentration were available in about $36 \%$ of the subjects. Baseline characteristics in each egg consumption category for the subjects with total cholesterol concentration data are shown in Table 2. An inverse 
Table 1. Baseline characteristics according to egg consumption categories* (Mean values and standard deviations)

\begin{tabular}{|c|c|c|c|c|c|c|c|c|c|}
\hline \multirow{2}{*}{$\begin{array}{l}\text { Egg consumption... } \\
\text { Baseline characteristics }\end{array}$} & \multicolumn{2}{|c|}{$\begin{array}{l}<1 \mathrm{~d} / \text { week } \\
(n 10491)\end{array}$} & \multicolumn{2}{|c|}{$\begin{array}{l}1-2 \mathrm{~d} / \text { week } \\
(n 20802)\end{array}$} & \multicolumn{2}{|c|}{$\begin{array}{c}3-4 \mathrm{~d} / \text { week } \\
(n 31182)\end{array}$} & \multicolumn{2}{|c|}{$\begin{array}{l}\text { Almost daily } \\
\text { (n 28260) }\end{array}$} & \multirow[b]{2}{*}{ Trend $P$} \\
\hline & Mean & SD & Mean & SD & Mean & SD & Mean & SD & \\
\hline Men (\%) & \multicolumn{2}{|c|}{$46 \cdot 5$} & \multicolumn{2}{|c|}{$50 \cdot 9$} & \multicolumn{2}{|c|}{$46 \cdot 6$} & \multicolumn{2}{|c|}{$47 \cdot 1$} & 0.0001 \\
\hline Age (years) & $55 \cdot 1$ & $8 \cdot 5$ & 51.9 & $7 \cdot 8$ & 51.9 & $7 \cdot 7$ & $52 \cdot 8$ & 7.9 & $<0.0001$ \\
\hline BMI $\left(\mathrm{kg} / \mathrm{m}^{2}\right)$ & $23 \cdot 7$ & $3 \cdot 2$ & $23 \cdot 6$ & $3 \cdot 1$ & 23.5 & $3 \cdot 1$ & 23.5 & $3 \cdot 2$ & $<0.0001$ \\
\hline Current smoker (\%) & \multicolumn{2}{|c|}{$28 \cdot 1$} & \multicolumn{2}{|c|}{30.5} & \multicolumn{2}{|c|}{$27 \cdot 0$} & \multicolumn{2}{|c|}{$26 \cdot 7$} & $<0.0001$ \\
\hline Drinker ( $\geq$ one per week) (\%) & \multicolumn{2}{|c|}{$32 \cdot 4$} & \multicolumn{2}{|c|}{38.6} & \multicolumn{2}{|c|}{37.5} & \multicolumn{2}{|c|}{$37 \cdot 8$} & $<0.0001$ \\
\hline Hypertension (\%) & \multicolumn{2}{|c|}{$17 \cdot 9$} & \multicolumn{2}{|c|}{$16 \cdot 0$} & \multicolumn{2}{|c|}{14.5} & \multicolumn{2}{|c|}{14.5} & $<0.0001$ \\
\hline Diabetes (\%) & \multicolumn{2}{|c|}{3.5} & \multicolumn{2}{|c|}{3.5} & \multicolumn{2}{|c|}{3.0} & \multicolumn{2}{|c|}{3.6} & 0.67 \\
\hline Use of cholesterol-lowering drugs (\%) & \multicolumn{2}{|c|}{$2 \cdot 7$} & \multicolumn{2}{|c|}{$2 \cdot 0$} & \multicolumn{2}{|c|}{1.6} & \multicolumn{2}{|c|}{1.2} & $<0.0001$ \\
\hline Cholesterol restriction† (\%) & \multicolumn{2}{|c|}{70.7} & \multicolumn{2}{|c|}{66.7} & \multicolumn{2}{|c|}{$66 \cdot 3$} & \multicolumn{2}{|c|}{$66 \cdot 2$} & $<0.0001$ \\
\hline Meat (d/week) & 4.9 & $1 \cdot 7$ & $5 \cdot 0$ & $2 \cdot 4$ & $5 \cdot 1$ & $2 \cdot 2$ & $5 \cdot 3$ & $2 \cdot 2$ & $<0.0001$ \\
\hline Fish (d/week) & 4.8 & $2 \cdot 2$ & $4 \cdot 8$ & $2 \cdot 1$ & $5 \cdot 2$ & $2 \cdot 0$ & $5 \cdot 4$ & $2 \cdot 0$ & $<0.0001$ \\
\hline Vegetables (d/week) & 4.4 & $2 \cdot 3$ & $5 \cdot 0$ & 1.9 & $5 \cdot 6$ & 1.7 & 5.9 & 1.6 & $<0.0001$ \\
\hline Fruits (d/week) & $3 \cdot 2$ & $2 \cdot 6$ & 3.5 & 2.5 & $4 \cdot 2$ & 2.5 & 4.5 & 2.5 & $<0.0001$ \\
\hline
\end{tabular}

${ }^{*}$ Cohort I (1990-2001) and cohort II (1993-2001) of Japan Public Health Center-based prospective study, combined. † Subjects who had an intention to restrict cholesterol-rich diets.

correlation between egg consumption and mean total cholesterol concentration as well as the frequency of the subjects with hypercholesterolaemia (those with total cholesterol concentration $\geq 2200 \mathrm{mg} / \mathrm{l}$ ), mean systolic blood pressures were noted (all trends $P<0.0001$ ). The other baseline characteristics were basically similar to those in Table 1 .

Egg consumption and coronary heart disease incidence - age and sex- and multivariate-adjusted Cox analyses

Altogether, there were 462 incidences of CHD during the mean follow-up of $10 \cdot 2$ years. Of them, 120 cases were fatal and 342 cases were non-fatal. Of the 120 cases of fatal
CHD, sixty-seven cases died within $1 \mathrm{~h}$ after the onset of symptoms.

The number of cases, their male percentage, person-years, age and sex-adjusted as well as multivariate-adjusted hazard ratios and their $95 \% \mathrm{CI}$ for $\mathrm{CHD}$ incidence according to egg consumption categories are shown in Table 3. The category of almost daily egg consumption was taken as the reference. There was no significant association between egg consumption and CHD incidence.

The secondary analyses in cohort I and II separately ( $P=0.23$ and $P=0.98$, respectively), in self-reported diabetic patients (trend $P=0.84$ ), analyses excluding subjects who had an intention to restrict cholesterol-rich diets (trend

Table 2. Baseline characteristics according to egg consumption categories in subjects with serum total cholesterol concentration data* (Mean values and standard deviations)

\begin{tabular}{|c|c|c|c|c|c|c|c|c|c|}
\hline \multirow{2}{*}{$\begin{array}{l}\text { Egg consumption... } \\
\text { Baseline characteristics }\end{array}$} & \multicolumn{2}{|c|}{$<1 \mathrm{~d} /$ week } & \multicolumn{2}{|c|}{$1-2 d /$ week } & \multicolumn{2}{|c|}{$3-4 d /$ week } & \multicolumn{2}{|c|}{ Almost daily } & \multirow[b]{2}{*}{ Trend $P$} \\
\hline & Mean & SD & Mean & SD & Mean & SD & Mean & SD & \\
\hline Subjects at risk† $(n)$ & \multicolumn{2}{|c|}{3633} & \multicolumn{2}{|c|}{6962} & \multicolumn{2}{|c|}{11542} & \multicolumn{2}{|c|}{10892} & \\
\hline Men $(\%)$ & \multicolumn{2}{|c|}{$32 \cdot 1$} & \multicolumn{2}{|c|}{$36 \cdot 8$} & \multicolumn{2}{|c|}{$34 \cdot 3$} & \multicolumn{2}{|c|}{$35 \cdot 8$} & 0.04 \\
\hline Age (years) & $58 \cdot 0$ & $8 \cdot 0$ & $54 \cdot 2$ & $8 \cdot 0$ & $53 \cdot 8$ & $7 \cdot 9$ & $54 \cdot 7$ & 7.9 & $<0.0001$ \\
\hline BMI $\left(\mathrm{kg} / \mathrm{m}^{2}\right)$ & 23.9 & $3 \cdot 3$ & 23.7 & $3 \cdot 1$ & $23 \cdot 6$ & $3 \cdot 0$ & 23.5 & $3 \cdot 3$ & $<0.0001$ \\
\hline Current smoker (\%) & \multicolumn{2}{|c|}{$16 \cdot 1$} & \multicolumn{2}{|c|}{$19 \cdot 8$} & \multicolumn{2}{|c|}{$17 \cdot 6$} & \multicolumn{2}{|c|}{$17 \cdot 3$} & 0.29 \\
\hline Drinker ( $\geq$ one per week) (\%) & \multicolumn{2}{|c|}{$23 \cdot 2$} & \multicolumn{2}{|c|}{$29 \cdot 9$} & \multicolumn{2}{|c|}{29.5} & \multicolumn{2}{|c|}{$30 \cdot 4$} & $<0.0001$ \\
\hline Systolic blood pressure $(\mathrm{mmHg})$ & 134 & 19 & 131 & 18 & 130 & 18 & 130 & 18 & $<0.0001$ \\
\hline Diastolic blood pressure $(\mathrm{mmHg})$ & 79 & 11 & 78 & 11 & 78 & 12 & 78 & 11 & $<0.0001$ \\
\hline Hypertension (\%) & \multicolumn{2}{|c|}{20.8} & \multicolumn{2}{|c|}{$17 \cdot 5$} & \multicolumn{2}{|c|}{$15 \cdot 2$} & \multicolumn{2}{|c|}{15.9} & $<0.0001$ \\
\hline Diabetes (\%) & \multicolumn{2}{|c|}{$2 \cdot 6$} & \multicolumn{2}{|c|}{3.0} & \multicolumn{2}{|c|}{$2 \cdot 4$} & \multicolumn{2}{|c|}{3.2} & $0 \cdot 16$ \\
\hline Total cholesterol (mg/l) & 2050 & 370 & 2030 & 360 & 2000 & 350 & 2000 & 340 & $<0.0001$ \\
\hline Total cholesterol $\geq 2200 \mathrm{mg} / \mathrm{l}(\%)$ & \multicolumn{2}{|c|}{33.5} & \multicolumn{2}{|c|}{29.7} & \multicolumn{2}{|c|}{$27 \cdot 5$} & \multicolumn{2}{|c|}{$27 \cdot 5$} & $<0.0001$ \\
\hline Use of cholesterol-lowering drugs (\%) & & & & & & & & & $<0.0001$ \\
\hline Cholesterol restriction $\ddagger(\%)$ & & & & & & & & & $<0.0001$ \\
\hline Meat (d/week) & $4 \cdot 8$ & $1 \cdot 7$ & 4.9 & 2.5 & $5 \cdot 0$ & $2 \cdot 3$ & $5 \cdot 2$ & $2 \cdot 2$ & $<0.0001$ \\
\hline Fish (d/week) & $5 \cdot 1$ & $2 \cdot 1$ & $5 \cdot 0$ & $2 \cdot 0$ & 5.5 & 1.9 & 5.7 & 1.8 & $<0.0001$ \\
\hline Vegetables (d/week) & $4 \cdot 8$ & $2 \cdot 3$ & $5 \cdot 3$ & 1.8 & $5 \cdot 8$ & 1.5 & $6 \cdot 1$ & 1.5 & $<0.0001$ \\
\hline Fruits (d/week) & 3.6 & $2 \cdot 7$ & $4 \cdot 0$ & 2.5 & $4 \cdot 6$ & $2 \cdot 4$ & 4.9 & 2.5 & $<0.0001$ \\
\hline
\end{tabular}

* Cohort I (1990-2001) and cohort II (1993-2001) of Japan Public Health Center-based prospective study, combined.

† Total number of subjects was 33029 .

Subjects who had an intention to restrict cholesterol-rich diets. 
Table 3. Coronary heart disease incidence according to egg consumption categories in the Japan Public Health Center-based prospective study (Hazard ratios (HR) and $95 \% \mathrm{Cl}$ )

\begin{tabular}{|c|c|c|c|c|c|c|c|c|c|}
\hline \multirow[b]{2}{*}{ Egg consumption... } & \multicolumn{2}{|c|}{$<1 \mathrm{~d} /$ week } & \multicolumn{2}{|c|}{$1-2 \mathrm{~d} /$ week } & \multicolumn{2}{|c|}{$3-4 \mathrm{~d} /$ week } & \multicolumn{2}{|c|}{ Almost daily } & \multirow[b]{2}{*}{ Trend $P$} \\
\hline & $\mathrm{HR}$ & $95 \% \mathrm{Cl}$ & $\mathrm{HR}$ & $95 \% \mathrm{Cl}$ & $\mathrm{HR}$ & $95 \% \mathrm{Cl}$ & $\mathrm{HR}$ & $95 \% \mathrm{Cl}$ & \\
\hline Subjects at risk ${ }^{*}(n)$ & \multicolumn{2}{|c|}{10491} & \multicolumn{2}{|c|}{20802} & \multicolumn{2}{|c|}{31182} & \multicolumn{2}{|c|}{28260} & \\
\hline Person-years & \multicolumn{2}{|c|}{96748} & \multicolumn{2}{|c|}{213907} & \multicolumn{2}{|c|}{323856} & \multicolumn{2}{|c|}{292858} & \\
\hline CHD incidence & & & & & & & & & \\
\hline Cases† $(n)$ & \multicolumn{2}{|c|}{64} & \multicolumn{2}{|c|}{110} & \multicolumn{2}{|c|}{147} & \multicolumn{2}{|c|}{141} & \\
\hline Men (\%) & \multicolumn{2}{|c|}{72} & \multicolumn{2}{|c|}{84} & \multicolumn{2}{|c|}{76} & \multicolumn{2}{|c|}{81} & \\
\hline Incidence (per 1000 person-years) & \multicolumn{2}{|c|}{0.66} & \multicolumn{2}{|c|}{0.51} & \multicolumn{2}{|c|}{0.45} & \multicolumn{2}{|c|}{0.48} & \\
\hline Age and sex-adjusted HR & $1 \cdot 28$ & $0.95,1.72$ & $1 \cdot 11$ & $0.86,1.42$ & 1.01 & $0.80,1 \cdot 27$ & 1 & - & 0.11 \\
\hline Multivariate-adjusted HR $\ddagger$ & $1 \cdot 19$ & $0.86,1.64$ & 1.00 & $0.77,1.30$ & 1.00 & $0.79,1.26$ & 1 & - & 0.45 \\
\hline
\end{tabular}

*Total number of subjects was 90735 .

† Subtotal of subjects was 462 .

Multivariate Cox analysis adjusted for age, sex, BMI, hypertension, diabetes, use of cholesterol-lowering drugs, smoking (never, ex-, and current smoker), alcohol

drinking (six categories), whether or not intended to avoid cholesterol-rich diets, consumption frequencies of meat, fish, vegetables, fruits, and cohort effects.

$P=0.45)$, analyses excluding those who were taking cholesterol-lowering drugs or those with total cholesterol concentration $\geq 2200 \mathrm{mg} / \mathrm{l}$ (trend $P=0.72$ ), analyses using quantitative variables (cholesterol and systolic blood pressure) in those who participated in a health check-up programme $(P=0.09)$, all yielded the basically similar results: there was no significant association between egg consumption and CHD incidence.

Total cholesterol concentration and coronary heart disease incidence - age and sex-adjusted and multivariate-adjusted Cox analyses

The number of cases, their male percentage, person-years, age and sex-adjusted as well as multivariate-adjusted hazard ratios and their $95 \% \mathrm{CI}$ for CHD incidence according to the total cholesterol concentration categories are shown in Table 4. The percentage of men progressively decreased in the groups with the higher total cholesterol concentration. Total cholesterol concentration was significantly related to CHD incidence (hazard ratio of CHD in those with total cholesterol concentration $\geq 2400 \mathrm{mg} / \mathrm{l}$ was $2 \cdot 17$ (95\% CI $1 \cdot 22,3.85$ ) as compared with those with total cholesterol concentration $<1800 \mathrm{mg} / \mathrm{l}$; trend $P=0.0018$ ).

\section{Discussion}

In the present study, we found that eating eggs more frequently, up to almost daily, was not associated with any consistent adverse effect on CHD incidence. We confirmed a positive association between total cholesterol concentration and CHD incidence in Japanese as in previous studies (Kodama et al. 1990; Kitamura et al. 1994; Okamura et al. 2003). We also found an inverse correlation between egg consumption and mean total cholesterol concentration as well as the frequency of the subjects with hypercholesterolaemia. The subjects with hypercholesterolaemia were more frequent among the groups of subjects who ate fewer eggs than those in the groups of subjects who ate more eggs. Controversies exist as to the relationship between dietary egg consumption and total cholesterol concentration. Some studies have shown no relationship between egg consumption and total cholesterol concentration (Mattson et al. 1972; Nichols et al. 1976a,b; Kummerow et al. 1977; Porter et al. 1977; Frank et al. 1978; Flynn et al. 1979; Dawber et al. 1982; Keys,

Table 4. Coronary heart disease incidence according to serum total cholesterol concentration categories in men and women (Japan Public Health Center-based prospective study) (Hazard ratios (HR) and $95 \% \mathrm{Cl}$ )

\begin{tabular}{|c|c|c|c|c|c|c|c|c|c|c|c|}
\hline \multirow{2}{*}{$\begin{array}{l}\text { Total cholesterol } \\
\text { concentration }(\mathrm{mg} / \mathrm{l}) \ldots\end{array}$} & \multicolumn{2}{|c|}{$<1800$} & \multicolumn{2}{|c|}{$1800-1999$} & \multicolumn{2}{|c|}{$2000-2199$} & \multicolumn{2}{|c|}{$2200-2399$} & \multicolumn{2}{|c|}{$\geq 2400$} & \multirow[b]{2}{*}{ Trend $P$} \\
\hline & $\mathrm{HR}$ & $95 \% \mathrm{Cl}$ & $\mathrm{HR}$ & $95 \% \mathrm{Cl}$ & $\mathrm{HR}$ & $95 \% \mathrm{Cl}$ & $\mathrm{HR}$ & $95 \% \mathrm{Cl}$ & $\mathrm{HR}$ & $95 \% \mathrm{Cl}$ & \\
\hline Subjects at risk ${ }^{*}(n)$ & \multicolumn{2}{|r|}{9162} & \multicolumn{2}{|r|}{7528} & \multicolumn{2}{|r|}{6896} & \multicolumn{2}{|r|}{4959} & \multicolumn{2}{|r|}{4484} & \\
\hline Men (\%) & \multicolumn{2}{|r|}{$45 \cdot 0$} & \multicolumn{2}{|r|}{$38 \cdot 0$} & \multicolumn{2}{|r|}{31.6} & \multicolumn{2}{|r|}{$28 \cdot 1$} & \multicolumn{2}{|r|}{$23 \cdot 3$} & $<0.0001$ \\
\hline Person-years & \multicolumn{2}{|c|}{96027} & \multicolumn{2}{|c|}{77847} & \multicolumn{2}{|c|}{70822} & \multicolumn{2}{|r|}{50438} & \multicolumn{2}{|c|}{45423} & \\
\hline \multicolumn{12}{|l|}{ CHD incidence } \\
\hline Cases† $(n)$ & \multicolumn{2}{|r|}{28} & \multicolumn{2}{|r|}{20} & \multicolumn{2}{|r|}{33} & \multicolumn{2}{|r|}{22} & \multicolumn{2}{|r|}{23} & \\
\hline Incidence (per 1000 person-years) & \multicolumn{2}{|r|}{$\begin{array}{c}79 \\
0.29\end{array}$} & \multicolumn{2}{|r|}{$\begin{array}{c}75 \\
0.26\end{array}$} & \multicolumn{2}{|r|}{0.47} & \multicolumn{2}{|r|}{0.44} & \multicolumn{2}{|r|}{0.51} & \\
\hline Age and sex-adjusted HR & 1 & - & 0.97 & $0.54,1.71$ & 1.93 & $1 \cdot 16,3 \cdot 20$ & 1.93 & $1 \cdot 10,3 \cdot 38$ & $2 \cdot 48$ & $1 \cdot 42,4.33$ & 0.0001 \\
\hline Multivariate-adjusted HR $\ddagger$ & 1 & - & 0.94 & $0.52,1.68$ & $1 \cdot 85$ & $1 \cdot 11,3 \cdot 10$ & 1.68 & $0.95,3.00$ & $2 \cdot 17$ & $1 \cdot 22,3 \cdot 85$ & 0.0018 \\
\hline
\end{tabular}

*Total number of subjects was 33029 .

† Subtotal of subjects was 126 .

¥ Multivariate Cox analysis adjusted for age, sex, BMI, systolic blood pressure, diabetes, use of cholesterol-lowering drugs or anti-hypertensive drugs, smoking (never, ex-, and current smoker), alcohol drinking (six categories), whether or not intended to avoid cholesterol-rich diets, consumption frequencies of egg, meat, fish, vegetables, fruits, and cohort effects. 
1984; Oh \& Miller, 1985), two studies have shown an inverse relationship (Tillotson et al. 1997; Song \& Kerver, 2000), and one found a positive relationship (Nakamura et al. 2004). As pointed out by Tillotson et al. (1997), the findings of the present study may be explained by the assumption that subjects with high total cholesterol concentration reduced their egg intake more than the others, and therefore we observed an inverse relationship between egg consumption and the frequency of hypercholesterolaemia. This assumption is supported by the trend among categories of egg consumption in the frequencies of intention to avoid taking a cholesterolrich diet. Therefore, a cause-effect reversal might have occurred between egg consumption and total cholesterol concentration.

Why are the results of the present study and those of the NIPPON DATA80 (Nakamura et al. 2004), with regard to the association between egg consumption and total cholesterol concentration, different? It is probably related to the difference in the year the two studies started. The NIPPON DATA80 study started in 1980, and the Japan Public Health Center-based study started in 1990 for cohort I and in 1993 for cohort II. In Japan, public awareness of total cholesterol concentration as one of the risk factors for atherosclerotic diseases was not remarkable until the mid 1980s. According to the National Nutritional Surveys, age-adjusted total cholesterol concentration increased from 1870 to $2020 \mathrm{mg} / \mathrm{l}$ for men and from 1900 to $2030 \mathrm{mg} / \mathrm{l}$ for women between 1980 and 1989 in Japan (Okayama et al. 1993). After the Health and Medical Service Law for the Elderly was enacted in 1983, all Japanese citizens aged 40 years and over had the opportunity to undergo screening for total cholesterol concentration, and those with hypercholesterolaemia were provided with health services such as health education or guidance to prevent coronary disease (Ministry of Health \& Welfare, 1987). Furthermore, cholestyramine appeared in the market in July 1985 and pravastatin in October 1989. The campaigns made by pharmaceutical companies to promote cholesterollowering drugs started to escalate, especially after the appearance of pravastatin in the market. Along with these movements, eggs became a popular media icon for the many of the dietary excesses of the population and the image for cholesterol, both dietary and serum (McNamara, 2000).

Eggs contain many other nutrients besides cholesterol, including unsaturated fats that may be beneficial in preventing CHD. Also, dietary cholesterol is not the principal factor affecting blood cholesterol concentration. The main dietary determinants of serum cholesterol are saturated fat and trans-fat intake. Therefore, restriction of egg consumption alone may not be sufficient for preventing CHD. However, in Japan, the reported per capita annual egg consumption in 1999 was 320, which was more than any other countries on the list (Bell, 2001), and previous reports showed that egg consumption made a greater contribution to total dietary cholesterol intake than in the USA (Ueshima et al. 1982; Okayama et al. 1995; Yoshida et al. 1998). Therefore, care for restricting egg consumption may be more important in Japan than in the other countries.

The major strengths of the present study include its prospective design, a general population with a high response rate $(80 \%)$, and the relatively low proportion of subjects lost to follow-up $(0.04 \%)$. The findings of the present study can be generalised to middle-aged and elderly Japanese men and women, because the study subjects were selected from the general population, and there was a high response rate. Moreover, two cohorts starting at different times produced the same results.

One of the limitations of the study was that we did not specify portion sizes on the food-frequency questionnaires, and thus we do not have reliable nutrient intake values. Therefore, we cannot use total energy intake as a covariate in the analyses. As we included dietary intake categories of meat, fish, vegetables, and fruits as covariates in the analyses, this limitation might have become less problematic. Second, total cholesterol concentration was available only in part of the subjects. However, the trend in the frequencies of intention to restrict taking cholesterol-rich diets was not different between subjects with or without the total cholesterol concentration data. This may imply that subjects with or without the total cholesterol concentration data were not different with regard to their health-conscious attitudes.

\section{Conclusion}

We found that eating eggs more frequently, up to almost daily, was not associated with any increase in CHD incidence. We found an inverse correlation between egg consumption and the frequency of hypercholesterolaemia in both sexes in both cohorts, probably because hypercholesterolaemic individuals avoided eating eggs.

\section{Acknowledgements}

The present study was supported by grants-in-aid for Cancer Research and for the Second Term Comprehensive Ten-Year Strategy for Cancer Control, and health sciences research grants (Research on Health Services, H16-019) from the Ministry of Health, Labor and Welfare of Japan. The authors thank all staff members in each study area and in the central office for their painstaking efforts to conduct the baseline survey and follow-up. Members of the Japan Public Health Centerbased Prospective Study (JPHC Study) Group are: S. Tsugane, T. Sobue, T. Hanaoka, M. Inoue, National Cancer Center; J. Ogata, S. Baba, T. Mannami, A. Okayama, National Cardiovascular Center; K. Miyakawa, F. Saito, A. Koizumi, Y. Sano, I. Hashimoto, Iwate Prefectural Ninohe Public Health Center; Y. Miyajima, N. Suzuki, S. Nagasawa, Y. Furusugi, Akita Prefectural Yokote Public Health Center; H. Sanada, Y. Hatayama, F. Kobayashi, H. Uchino, Y. Shirai, T. Kondo, R. Sasaki, Y. Watanabe, Nagano Prefectural Saku Public Health Center; Y. Kishimoto, E. Tanaka, M. Kinjo, T. Fukuyama, M. Irei, Okinawa Prefectural Chubu Public Health Center; K. Imoto, H. Yazawa, T. Seo, A. Seiko, F. Ito, Katsushika Public Health Center; A. Murata, K. Minato, K. Motegi, T. Fujieda, Ibaraki Prefectural Mito Public Health Center; K. Matsui, T. Abe, M. Katagiri, Niigata Prefectural Kashiwazaki Public Health Center; M. Doi, Y. Ishikawa, A. Terao, Kochi Prefectural Chuo-higashi Public Health Center; H. Sueta, H. Doi, M. Urata, F. Ide, Nagasaki Prefectural Kamigoto Public Health Center; H. Sakiyama, N. Onga, H. Takaesu, Okinawa Prefectural Miyako Public Health Center; F. Horii, I. 
Asano, H. Yamaguchi, K. Aoki, S. Maruyama, M. Ichii, Osaka Prefectural Suita Public Health Center; S. Matsushima, S. Natsukawa, Saku General Hospital; S. Watanabe, M. Akabane, Tokyo University of Agriculture; M. Konishi, K. Okada, Ehime University; H. Iso, Y. Honda, Tsukuba University; H. Sugimura, Hamamatsu University School of Medicine; Y. Tsubono, Tohoku University; N. Kabuto, National Institute for Environmental Studies; S. Tominaga, Aichi Cancer Center; M. Iida, W. Ajiki, Osaka Medical Center for Cancer and Cardiovascular Disease; S. Sato, Osaka Medical Center for Health Science and Promotion; N. Yasuda, Kochi Medical School; S. Kono, Kyushu University; K. Suzuki, Research Institute for Brain and Blood Vessels Akita; Y. Takashima, Kyorin University; E. Maruyama, Kobe University; M. Yamaguchi, Y. Matsumura, S. Sasaki, National Institute of Health and Nutrition; T. Kadowaki, University of Tokyo.

\section{References}

Bell D (2001) Per Capita Egg Consumption by Country 1999 and 2000. http://animalscience.ucdavis.edu/Avian/PerCapitaEggConsumption byCountry2000.htm

Cox DR (1972) Regression models and life-tables. J R Stat Soc 34, B187-B220

Dawber TR, Nickerson RJ, Brand FN \& Pool J (1982) Eggs, serum cholesterol, and coronary heart disease. Am J Clin Nutr 36, $617-625$

Expert Panel on Detection, Evaluation and Treatment of High Blood Cholesterol in Adults (2001) Executive Summary of the Third Report of the National Cholesterol Education Program (NCEP) Expert Panel on Detection, Evaluation, and Treatment of High Blood Cholesterol in Adults (Adult Treatment Panel III). JAMA 285, 2486-2489.

Flynn MA, Nolph GB, Flynn TC, Kahrs R \& Krause G (1979) Effect of dietary egg on human serum cholesterol and triglycerides. Am J Clin Nutr 32, 1051-1057.

Frank GC, Berenson GS \& Webber LS (1978) Dietary studies and the relationship of diet to cardiovascular disease risk factor variables in 10-year-old children - The Bogalusa Heart Study. Am J Clin Nutr 31, 328-340.

Hu FB, Stampfer MJ, Rimm EB, et al. (1999) A prospective study of egg consumption and risk of cardiovascular disease in men and women. JAMA 281, 1387-1394.

Ishihara J, Sobue T, Yamamoto S, Yoshimi I, Sasaki S, Kobayashi M, Takahashi T, Iitoi Y, Akabane M \& Tsugane S (2003) Validity and reproducibility of a self-administered food frequency questionnaire in the JPHC Study Cohort II: study design, participant profile and results in comparison with Cohort I. J Epidemiol 13, Suppl. 1, S134-S147.

Iso $\mathrm{H}$, Baba $\mathrm{S}$, Mannami $\mathrm{T}$, Sasaki S, Okada K, Konishi M \& Tsugane S (2004) Alcohol consumption and risk of stroke among middle-aged men: the JPHC Study Cohort I. Stroke 35, $1124-1129$.

Keys A (1984) Serum cholesterol response to dietary cholesterol. Am J Clin Nutr 40, 351-359.

Kitamura A, Iso H, Naito Y, Iida M, Konishi M, Folsom AR, Sato S, Kiyama M, Nakamura M \& Sankai T (1994) High-density lipoprotein cholesterol and premature coronary heart disease in urban Japanese men. Circulation 89, 2533-2539.

Kodama K, Sasaki H \& Shimizu Y (1990) Trend of coronary heart disease and its relationship to risk factors in a Japanese population: a 26-year follow-up, Hiroshima/Nagasaki study. Jpn Circ J 54, 414-421.

Kummerow FA, Kim Y, Hull J, Pollard J, Ilinov P, Drossiev DL \& Valek J (1977) The influence of egg consumption on the serum cholesterol level in human subjects. Am J Clin Nutr 30 , 664-673.

McNamara DJ (2000) The impact of egg limitations on coronary heart disease risk: do the numbers add up? J Am Coll Nutr 19, Suppl. 5, 540S-548S.

Mattson FH, Erickson BA \& Kligman AM (1972) Effect of dietary cholesterol on serum cholesterol in man. Am J Clin Nutr 25, 589-594.

Ministry of Health and Welfare (1987) Manual for Health Examination under Health and Medical Service Law for the Elderly (in Japanese)Tokyo: Japan Public Health Association.

Myers GL, Kimberly MM, Waymack PP, Smith SJ, Cooper GR \& Sampson EJ (2000) A reference method laboratory network for cholesterol: a model for standardization and improvement of clinical laboratory measurements. Clin Chem 46, 1762-1772.

Nakamura M, Sato S \& Shimamoto T (2003) Improvement in Japanese clinical laboratory measurements of total cholesterol and HDL-cholesterol by the US Cholesterol Reference Method Laboratory Network. J Atheroscler Thromb 10, 145-153.

Nakamura Y, Okamura T, Tamaki S, Kadowaki T, Hayakawa T, Kita Y, Okayama A \& Ueshima H (2004) Egg consumption, serum cholesterol, and cause-specific and all-cause mortality: NIPPON DATA80, 1980-94. Am J Clin Nutr 80, 58-63.

Nichols AB, Ravenscroft C, Lamphiear DE \& Ostrander LD Jr (1976a) Daily nutritional intake and serum lipid concentrations. The Tecumseh study. Am J Clin Nutr 29, 1384-1392.

Nichols AB, Ravenscroft C, Lamphiear DE \& Ostrander LD Jr (1976b) Independence of serum lipid concentrations and dietary habits. The Tecumseh study. JAMA 236, 1948-1953.

Oh SY \& Miller LT (1985) Effect of dietary egg on variability of plasma cholesterol levels and lipoprotein cholesterol. Am J Clin Nutr 42, 421-431.

Okamura T, Kadowaki T, Hayakawa T, Kita Y, Okayama A \& Ueshima H (2003) What cause of mortality can we predict by cholesterol screening in the Japanese general population? J Intern Med 253, 169-180.

Okayama A, Ueshima H, Marmot MG, Elliott P, Yamakawa M \& Kita Y (1995) Different trends in serum cholesterol levels among rural and urban populations aged 40-59 in Japan from 1960 to 1990. J Clin Epidemiol 48, 329-337.

Okayama A, Ueshima H, Marmot MG, Nakamura M, Kita Y \& Yamakawa M (1993) Changes in total serum cholesterol and other risk factors for cardiovascular disease in Japan, 1980-1989. Int J Epidemiology 22, 1038-1047.

Porter MW, Yamanaka W, Carlson SD \& Flynn MA (1977) Effect of dietary egg on serum cholesterol and triglyceride of human males. Am J Clin Nutr 30, 490-495.

Sobue T, Yamamoto S \& Watanabe S (2001) Smoking and drinking habits among the JPHC study participants at baseline survey. $J$ Epidemiol 11, Suppl. 6, S44-S56.

Song WO \& Kerver JM (2000) Nutritional contribution of eggs to American diets. J Am Coll Nutr 19, 556S-562S.

Tillotson JL, Bartsch GE, Gorder D, Grandits GA \& Stamler J (1997) Food group and nutrient intakes at baseline in the Multiple Risk Factor Intervention Trial. Am J Clin Nutr 65, Suppl. 1, 228S-257S.

Tsubono Y, Kobayashi M, Sasaki S \& Tsugane S (2003) Validity and reproducibility of a self-administered food frequency questionnaire used in the baseline survey of the JPHC Study Cohort I. J Epidemiol 13, Suppl. 6, S125-S133.

Tsugane S, Sasaki S, Kobayashi M, Tsubono Y \& Sobue T (2001) Dietary habits among the JPHC study participants at baseline survey. J Epidemiol 11, Suppl., S30-S43.

Tsugane S \& Sobue T (2001) Baseline survey of JPHC study design and participation rate. J Epidemiol 11, Suppl. 6, S24-S29.

Tunstall-Pedoe H, Kuulasmmaa K, Amouyel P, Arveiler D, Rajakangas AM \& Pajak A (1994) Myocardial infarction and coronary deaths in the World Health Organization MONICA 
Project. Registration procedures, event rates, and case-fatality rates in 38 populations from 21 countries in four continents. Circulation 90, 583-612.

Ueshima H, lida M, Shimamoto T, Konishi M, Tanigaki M, Doi M, Nakanishi N, Takayama Y, Ozawa H \& Komachi Y (1982) Dietary intake and serum total cholesterol level: their relationship to different lifestyles in several Japanese populations. Circulation 66, 519-526.
Watanabe S, Tsugane S, Sobue T, Konishi M \& Baba S (2001) Study design and organization of the JPHC study. J Epidemiol 11, Suppl. 6, S3-S7.

Yoshida Y, Okayama A, Mikawa K \& Ueshima H (1998) Dietary intake and its relationship to serum cholesterol concentrations among three Japanese populations in the early 1990s: INTERSALT II study in Japan. J Shiga Univ Med Sci 13, 63-79. 